\title{
Clinical and Etiological Characteristics of Epilepsy in the Elderly: A Hospital-Based Study from a Tertiary Care Referral Center of Niamey, Niger
}

\author{
Hamid Assadeck ${ }^{1,2}$ Moussa Toudou-Daouda ${ }^{1}$ Zakaria Mamadou ${ }^{1}$ Mahadi Moussa-Konate ${ }^{3}$ \\ Fatimata Hassane-Djibo ${ }^{1}$ Dijbo Douma-Maiga ${ }^{2}$
}

${ }^{1}$ Department of Neurology, National Hospital of Niamey, Niamey, Address for correspondence Moussa Toudou-Daouda, MD, Niger

2Department of Medicine and Medical Specialties, Faculty of Medicine and Pharmacy, Abdou Moumouni University, Niamey, Niger

${ }^{3}$ Department of Neurology, General Reference Hospital of Niamey, Niamey, Niger Department of Neurology, National Hospital of Niamey, PO Box 238, Niamey, Niger (e-mail: moussatoudou@gmail.com).

J Neurosci Rural Pract 2019;10:571-575

\begin{abstract}
Keywords

- elderly

- epilepsy

- hospital-based study

- Niamey

- Niger

- sub-Saharan Africa
\end{abstract}

Objectives The aim of this study is to evaluate the management of epilepsy in the elderly at a tertiary referral center in Niger to obtain a comprehensive understanding to determine the intrahospital deficiencies to improve and to make recommendations in terms to improve the management of epilepsy in the elderly in Niger.

Materials and Methods We conducted a retrospective study at the Neurology Outpatient Clinic of the National Hospital of Niamey (Niger) over a period of 5 years from May 2013 to May 2018, collecting all cases of patients aged 60 years or over diagnosed with epilepsy by neurologists. From the registers of consultation, we collected and analyzed for each patient the demographic, clinical, etiological, and therapeutic data, as well as the outcomes during follow-up visits.

Results Of the 4,576 patients of all ages seen during the period of our study, we included 62 patients aged 60 years or over diagnosed with epilepsy with a hospital frequency of $1.35 \%$. The mean age of patients was $65.82 \pm 5.72$ years (range: 60 and 83 years) with a predominance of the male sex (sex ratio at 1.6). Patients aged 60 to 64 years were the most represented (43.5\%). Generalized tonic-clonic seizures were the most frequent (41.9\%), followed by focal to bilateral tonic-clonic seizures (25.8\%). All patients underwent electroencephalogram. Only 30 patients $(48.4 \%)$ underwent brain imaging, and mainly brain computed tomography scan. The etiologies included poststroke epilepsy $(25.8 \%)$, brain tumors $(3.2 \%)$, cerebral toxoplasmosis $(3.2 \%)$, and cerebral meningioma (1.6\%). We found 41 cases $(66.1 \%$ ) of epilepsy without definite etiology and with an incomplete workup. Carbamazepine and phenobarbital were the only two antiepileptic drugs (AEDs) used.

Conclusion The present study shows limited access to newer generation AEDs and diagnostic tests of epilepsy in Niger. Considerable efforts should be made to facilitate for people living with epilepsy the accessibility to diagnostic tests and the newer generation AEDs to improve the quality of epilepsy management in Niger.
DOI https://doi.org/

$10.1055 / \mathrm{s}-0039-1700308$

ISSN 0976-3147.
C2019 Association for Helping Neurosurgical Sick People
License terms

()(1) $\Theta \circledast$ 


\section{Introduction}

Epilepsy is one of the most common chronic neurological diseases affecting $>50$ million people worldwide of all ages regardless of gender, and regardless of their geographic and ethnic origin. ${ }^{1}$ It constitutes a major public health concern in the world, particularly in developing countries, especially in sub-Saharan African countries. Nearly $80 \%$ of people worldwide living with epilepsy reside in developing countries. ${ }^{1}$ The incidence of epilepsy increases substantially with age and peaks after the age of 80 years. ${ }^{2}$ Epilepsy is the third neurological condition in the elderly following stroke and dementia. ${ }^{3}$ Nowadays, the proportion of the elderly in sub-Saharan Africa increases progressively because of the improvement in the quality of health care and consequently, an augmentation of cardiovascular, cerebrovascular, and neurodegenerative diseases and neoplasms, which increase the incidence of epilepsy in the elderly. Only a few studies had been published on epilepsy in the elderly in sub-Saharan Africa. ${ }^{4-6}$ In Niger, there are no epidemiological data on epilepsy in the elderly. Thus, we designed this study to evaluate the management of epilepsy in the elderly at a tertiary referral center in Niger to obtain a comprehensive understanding to determine the intrahospital deficiencies to improve and to make recommendations in terms to improve the management of epilepsy in the elderly in Niger.

\section{Materials and Methods}

\section{Study Design}

We retrospectively collected from the registers of consultation all patients aged 60 years or over diagnosed with epilepsy by Neurologists at the Neurology Outpatient Clinic of the National Hospital of Niamey (Niger) over a period of 5 years from May 2013 to May 2018. Until the beginning of the year 2018, this hospital was the sole largest urban and tertiary care referral center in Niger that had neurologists. People living with neurological diseases were generally referred to this referral center for specialized care before the year 2018. This hospital covers an area of 23,120.50 $\mathrm{m}^{2}$ and comprises 36 buildings with a bed capacity of 790 . Until this day, it attracts people from all corners of the country to seek medical care in various medical fields.

The study was approved by the Institutional Review Board of the Faculty of Medicine of Abdou Moumouni University of Niamey (Niger) in accordance with the Declaration of Helsinki.

\section{Patients}

The study included all patients aged 60 years or over that consulted at the Neurology Outpatient Clinic of the National Hospital of Niamey (Niger) for epilepsy. The diagnosis of epilepsy was made for all patients by neurologists according to the 2014 revised definition of epilepsy of International League Against Epilepsy (ILAE) official report: at least two unprovoked (or reflex) seizures occurring $>24$ hours apart or one unprovoked (or reflex) seizure in individuals who have risk factors such as cerebrovascular disease, central nervous system infection, and traumatic brain injury. ${ }^{7}$ For each patient, we collected from the registers of consultation the following information: age of diagnosis, gender, medical history, type of seizure, type of epilepsy, and antiepileptic treatment. All patients underwent electroencephalogram (EEG). Brain imaging (magnetic resonance imaging [MRI] or computed tomography [CT] scan) had not been performed in many patients because of limited access. Only 30 patients (48.4\%) underwent brain imaging, and mainly CT scan of the brain. All patients received laboratory tests including standard biochemistry (fasting blood glucose, creatinine, urea, sodium, potassium, C-reactive protein, and transaminases), blood count, erythrocyte sedimentation rate, and serological tests for human immunodeficiency virus, syphilis, hepatitis B, and hepatitis C. Systemic immunological tests (soluble antinuclear antigen antibodies, antinuclear antibodies, anti-double-stranded deoxyribonucleic acid, etc.) and measurement of antineuronal antibodies were not performed. All patients were followed and evaluated during the period of the study by neurologists. The response to antiepileptic treatment was evaluated during the follow-up visits. We considered a patient to have poor drug compliance when epileptic seizures are well controlled in case of regular drug intake and then in case of recrudescence of epileptic seizures when drug intake is imperfect or irregular (drug intake jump).

\section{Statistical Analysis}

In the descriptive analysis of the data, patient characteristics were expressed as percentages for the qualitative variables and mean \pm standard deviation for the quantitative variables. The chi-squared test of Pearson was used to compare the proportions of the qualitative variables. The Student's $t$-test was used to compare two observed means. To examine the difference between several groups, we used one-way analysis of variation. Values of $p<0.05$ were considered as statistically significant. All statistical analyses were performed with SPSS software version 20.0 (SPSS Inc., Armonk, New York, United States).

\section{Results}

\section{Demographic Characteristics}

During the period of the study, 4,576 patients of all ages consulted at the Neurology Outpatient Clinic of National Hospital of Niamey (Niger), among whom 62 patients aged 60 years or over had epilepsy with a hospital frequency of 1.35\%. - Table 1 summarizes the demographic characteristics of the 62 patients. We included 38 men and 24 women with males-to-females ratio of 1.6. The patients were aged 60 to 83 years with a mean age of $65.82 \pm 5.72$ years. Patients aged 60 to 64 years were the most represented (43.5\%). Cerebrovascular disease was the main medical history of patients (21\%). We found no statistically significant difference between the mean age of men and women (66.16 \pm 6.25 years for men vs. $65.29 \pm 4.83$ years for women, $p=0.56$ ).

\section{Clinical Characteristics}

Generalized tonic-clonic seizures were the most frequent (41.9\%) followed by focal to bilateral tonic-clonic seizures 
Table 1 Demographic characteristics of patients $(n=62)$

\begin{tabular}{|c|c|}
\hline Variables & $n(\%)$ \\
\hline \multicolumn{2}{|l|}{ Sex } \\
\hline Males & $38(61.3)$ \\
\hline Females & $24(38.7)$ \\
\hline Sex ratio (males/females) & 1.6 \\
\hline \multicolumn{2}{|l|}{ Age (years) } \\
\hline Mean & $65.82 \pm 5.72$ \\
\hline Range & $60-83$ \\
\hline Mean/males & $66.16 \pm 6.25$ \\
\hline Mean/females & $65.29 \pm 4.83$ \\
\hline $60-64$ & $27(43.5)$ \\
\hline $65-69$ & $16(25.8)$ \\
\hline $70-74$ & $14(22.6)$ \\
\hline$>75$ & $5(8.1)$ \\
\hline \multicolumn{2}{|l|}{ Past medical history } \\
\hline Human immunodeficiency virus infection & $1(1.6)$ \\
\hline Arterial hypertension & $2(3.2)$ \\
\hline $\begin{array}{l}\text { Arterial hypertension associated with } \\
\text { diabetes }\end{array}$ & $1(1.6)$ \\
\hline Cerebrovascular disease & $13(21)$ \\
\hline Cerebral toxoplasmosis & $1(1.6)$ \\
\hline None & $44(71)$ \\
\hline
\end{tabular}

(25.8\%) (-Table 2). Hemiparesis and aphasia were the two associated clinical signs. Generalized tonic-clonic seizures and focal to bilateral tonic-clonic seizures were the most frequent in patients aged 60 to 64 years (-Table $\mathbf{3}$ ). We found no statistically significant difference between the mean age of patients with focal epilepsies and those with generalized epilepsies ( $67 \pm 6.59$ years versus $64.39 \pm 4.1$ years; $p=0.074$ ).

\section{Etiologies}

An etiology was identified only in about one-third of patients (33.9\%) (-Table 2). Among the identified etiologies, cerebrovascular disease was the most common. The other twothird of patients had no definite etiology, and the workup was incomplete. Only 30 patients (48.4\%) underwent brain imaging and was normal in nine patients (14.5\%). No patient received the measurement of antineuronal antibodies for autoimmune encephalitis. Poststroke epilepsy was the most frequent in patients aged 60 to 64 years ( - Table 3 ).

\section{Therapeutic Characteristics and Outcomes during Follow-Up Visits}

Carbamazepine ( $\mathrm{CBZ}$ ) and phenobarbital $(\mathrm{PB})$ were the only two molecules used in our patients (-Table 2). Only two patients (3.2\%) had received a dual therapy associating CBZ and $\mathrm{PB}$.

We did not have any cases of drug resistance in this study. However, we found 18 patients (29\%) with poor drug compliance with persistent epileptic seizures. All 18 patients had no established etiological diagnosis, and the workup was incomplete.
Table 2 Clinical, etiological, and therapeutic characteristics as well as the outcomes of patients $(n=62)$

\begin{tabular}{|c|c|}
\hline Variables & $n(\%)$ \\
\hline \multicolumn{2}{|l|}{ Seizure types } \\
\hline Generalized myoclonic seizures & $2(3.2)$ \\
\hline Generalized tonic-clonic seizures & $26(41.9)$ \\
\hline Focal aware seizures & $12(19.4)$ \\
\hline Focal impaired awareness seizures & $6(9.7)$ \\
\hline Focal to bilateral tonic-clonic seizures & $16(25.8)$ \\
\hline \multicolumn{2}{|l|}{ Associated clinical signs } \\
\hline Hemiparesis & $2(3.2)$ \\
\hline Aphasia & $1(1.6)$ \\
\hline \multicolumn{2}{|l|}{ Clinical diagnoses of epilepsy } \\
\hline Generalized epilepsies & $28(45.1)$ \\
\hline Focal epilepsies & $34(54.9)$ \\
\hline \multicolumn{2}{|c|}{ Electroencephalographic diagnoses of epilepsy } \\
\hline Generalized epilepsies & $22(35.5)$ \\
\hline Focal epilepsies & $29(46.8)$ \\
\hline Normal electroencephalogram & $11(17.7)$ \\
\hline \multicolumn{2}{|l|}{ Etiologies } \\
\hline Cerebral meningioma & $1(1.6)$ \\
\hline Poststroke epilepsy & $16(25.8)$ \\
\hline Cerebral toxoplasmosis & $2(3.2)$ \\
\hline Brain tumor & $2(3.2)$ \\
\hline Not determined & $41(66.1)$ \\
\hline \multicolumn{2}{|l|}{ Antiepileptic drugs } \\
\hline Carbamazepine & $45(72.6)$ \\
\hline Phenobarbital & $15(24.2)$ \\
\hline Carbamazepine + phenobarbital & $2(3.2)$ \\
\hline \multicolumn{2}{|l|}{ Outcomes during follow-up visits } \\
\hline Seizure control & $44(71)$ \\
\hline Poor drug compliance & $18(29)$ \\
\hline Death & $3(4.8)$ \\
\hline Stroke recurrence & $2(2.6)$ \\
\hline Memory disorders & $1(1.6)$ \\
\hline
\end{tabular}

Two patients presented stroke recurrence. These patients were aged 60 to 64 years (-Table 3 ). We recorded three deaths among which one patient had died following a stroke recurrence.

\section{Discussion}

In this 5-year retrospective study, we evaluate the management of epilepsy in the elderly at a tertiary referral center in Niger to obtain a comprehensive understanding to determine the intrahospital deficiencies to improve and to make recommendations in terms to improve the management of epilepsy in the elderly in Niger. The age of 60 years or over is used to define the elderly in Niger. To remind, the life expectancy at birth of people from Niger is 61.8 years, and people aged 
Table 3 Demographic, clinical, etiological, and therapeutic characteristics as well as the outcomes of the patients by age group

\begin{tabular}{|c|c|c|c|c|c|}
\hline \multirow[t]{2}{*}{ Variables } & \multicolumn{4}{|c|}{ Age group } & \multirow[t]{2}{*}{ Tota } \\
\hline & $60-64$ & $65-69$ & $70-74$ & $>75$ & \\
\hline \multicolumn{6}{|l|}{ Sex } \\
\hline Male & 16 & 11 & 7 & 4 & 38 \\
\hline Female & 11 & 5 & 7 & 1 & 24 \\
\hline \multicolumn{6}{|l|}{ Past medical history } \\
\hline Human immunodeficiency virus infection & 1 & 0 & 0 & 0 & 1 \\
\hline Arterial hypertension & 1 & 1 & 0 & 0 & 2 \\
\hline Arterial hypertension associated with diabetes & 1 & 0 & 0 & 0 & 1 \\
\hline Cerebrovascular disease & 5 & 4 & 2 & 2 & 13 \\
\hline Cerebral toxoplasmosis & 1 & 0 & 0 & 0 & 1 \\
\hline \multicolumn{6}{|l|}{ Seizure types } \\
\hline Generalized myoclonic seizures & 2 & 0 & 0 & 0 & 2 \\
\hline Generalized tonic-clonic seizures & 13 & 7 & 6 & 0 & 26 \\
\hline Focal aware seizures & 3 & 4 & 3 & 2 & 12 \\
\hline Focal impaired awareness seizures & 0 & 1 & 3 & 2 & 6 \\
\hline Focal to bilateral tonic-clonic seizures & 9 & 4 & 2 & 1 & 16 \\
\hline \multicolumn{6}{|l|}{ Etiologies } \\
\hline Cerebral meningioma & 1 & 0 & 0 & 0 & 1 \\
\hline Poststroke epilepsy & 6 & 5 & 3 & 2 & 16 \\
\hline Cerebral toxoplasmosis & 1 & 1 & 0 & 0 & 2 \\
\hline Brain tumor & 2 & 0 & 0 & 0 & 2 \\
\hline Not determined & 17 & 10 & 11 & 3 & 41 \\
\hline \multicolumn{6}{|l|}{ Antiepileptic drugs } \\
\hline Carbamazepine & 19 & 11 & 12 & 3 & 45 \\
\hline Phenobarbital & 8 & 4 & 1 & 2 & 15 \\
\hline Carbamazepine + phenobarbital & 0 & 1 & 1 & 0 & 2 \\
\hline \multicolumn{6}{|l|}{ Outcomes during follow-up visits } \\
\hline Seizure control & 21 & 10 & 10 & 3 & 44 \\
\hline Poor drug compliance & 6 & 6 & 4 & 2 & 18 \\
\hline Death & 2 & 0 & 1 & 0 & 3 \\
\hline Stroke recurrence & 2 & 0 & 0 & 0 & 2 \\
\hline Memory disorders & 0 & 0 & 1 & 0 & 1 \\
\hline
\end{tabular}

60 years or over represent only $4.2 \%$ of the general population. ${ }^{8}$ We considered consequently in this study a patient as an elderly any patient aged 60 years or over. We study only the people aged 60 years or over living with epilepsy which consulted at the Neurology Outpatient Clinic of the National Hospital of Niamey (Niger).

The hospital frequency of epilepsy in the elderly in the present study was $1.35 \%$ that appeared to be low. KuateTegueu et al $^{5}$ reported in 2015 in two Urban Health Centers of Douala (Cameroon) a hospital frequency of patients aged 60 or over of $3.7 \%$ that is twice higher to that observed in our study. In a Malian hospital-based study in $2013,{ }^{4}$ the hospital frequency of patients aged 60 or over was $1.31 \%(23 / 1,753)$ that is similar to our findings. In a door-to-door survey in Ecuador, Placencia et $\mathrm{al}^{9}$ reported a prevalence of epilepsy in the people aged 60 years or over of $1.27 \%$ o for all ages
$(92 / 72,121)$ and $15.3 \%$ o $(92 / 5,990)$ for ages superior or equal to 60 years. This observed difference could be explained by the fact that the methodology and the design are different between these studies.

We noted in this study a predominance of focal-onset seizures (54.9\%). This predominance of focal-onset seizures has been reported previously in several studies.,6,10,11 However, Kuate-Tegueu et $\mathrm{al}^{5}$ reported as far as they are concerned in Cameroon a predominance of generalized tonic-clonic seizures (44\%).The high prevalence of focal brain lesions in the elderly explains this predominance of focal-onset seizures in these persons.

Only 30 patients (48.4\%) had received brain imaging in this study because of its limited accessibility for the majority of patients. Among the identified etiologies of epilepsy in this study, cerebrovascular disease was the most common. 
This predominance of cerebrovascular disease among the causes of epilepsy in the elderly has been reported previously in several studies..$^{4-6,12,13}$ In 41 patients (66.1\%), we did not find a definite cause. Of these 41 patients, only nine patients underwent brain imaging that was normal. Maiga et $\mathrm{al}^{4}$ in their study reported a proportion of epilepsies without a defined etiology of $18 \%$, but these authors do not specify if the workup was complete or no in their patients. Chen et $\mathrm{a}^{10}$ reported as far as they are concerned autoimmune encephalitis as the most common identified causes followed by cerebrovascular disease. The same authors report a proportion of epilepsies without a defined etiology of $21.4 \%$. In this study, the high proportion of the patients without defined etiology demonstrates the problem of the availability and accessibility of complementary examinations in the management of epilepsy in Niger.

We used only the first-generation antiepileptic drugs (AEDs) in our study (CBZ and PB). Similar results have been reported in previous studies in sub-Saharan Africa on epilepsy in the elderly. ${ }^{4,5}$ However, Chen et $\mathrm{al}^{10}$ reported, as far as they are concerned, the preferential prescription of newer generation AEDs (levetiracetam and oxcarbazepine). Newer generation AEDs are not only inaccessible in Niger, but also they are expensive for patients, which the majority of them do not have a source of monthly income. In addition, the prescription of these AEDs would increase the rate of poor drug compliance.

Our study has some limitations. First, as the study originates from a single center, the generalization of our findings may be limited. Second, the retrospective nature of the study explains why some details were not provided, especially profession, provenance (rural or urban), socioeconomic status, marital status, cultures, etc. Third, limited access to complementary examinations such as MRI or antineuronal antibodies explaining the high prevalence of epilepsies without definite etiology. Fourth, we did not compare in the study the group of the elderly with epilepsy to that of the elderly without epilepsy.

\section{Conclusion}

The study provides a description of demographic, clinical, and etiological characteristics of epilepsy in hospital elderly from Niger. These hospital findings permit to create a database on epilepsy in the elderly that will provide data for comparison with data of future studies on epilepsy in Niger. The study shows limited access to newer generation AEDs and diagnostic tests of epilepsy in Niger. Considerable efforts should be made in Niger, particularly at the National Hospital of Niamey to facilitate for people living with epilepsy the accessibility to diagnostic tests and newer generation AEDs to improve the quality of epilepsy management in Niger. A prospective multicenter study is needed in Niger to determine the exact prevalence of elderly living with epilepsy and to improve the management of these patients.

\author{
Funding \\ None. \\ Conflict of Interest \\ None declared.
}

\section{References}

1 World Health Organization. Epilepsy Fact Sheet. Available from: https://www.who.int/en/news-room/fact-sheets/detail/ epilepsy. [Last accessed on 2019 Feb 13; Last update on 2019 Feb 07]

2 Hauser WA. Seizure disorders: the changes with age. Epilepsia 1992;33(Suppl 4):S6-S14

3 Ziso B, Dixon PA, Marson AG. Epilepsy management in older people: lessons from National Audit of Seizure management in Hospitals (NASH) Seizure 2017;50:33-37

4 Maiga Y, Daou M, Kuate C, et al. Epilepsy in the elderly: experience of neurology service of the Gabriel Toure Hospital in Bamako, Mali. Afr Middle East Epilepsy J 2013;2:27-30

5 Kuate-Tegueu C, Doumbe J, Kammegne-Younang EJ, Noubissi G, Djientcheu VP. Epilepsy of the elderly: epidemiological aspects in two urban health centers of Douala (Cameroon). Health Sci Dis 2015;16:1-6

6 Morel Y, Cisse FA, Drame F, Cisse A, Guilavogui V, Sory Souare I. Épilepsie du sujet âgé à Conakry. Rev Neurol (Paris) 2012;168:A65

7 Fisher RS, Acevedo C, Arzimanoglou A, et al. ILAE official report: a practical clinical definition of epilepsy. Epilepsia 2014;55(4):475-482

8 Organization Mondiale de la Santé. OMS Stratégie de Coopération: Un Aperçu: Niger. Organization Mondiale de la Santé; 2018. Available from: http://www.who.int/iris/ handle/10665/136784. [Last visited on 2018 Dec 25]

9 Placencia M, Shorvon SD, Paredes V, et al. Epileptic seizures in an Andean region of Ecuador. Incidence and prevalence and regional variation. Brain 1992;115(Pt 3):771-782

10 Chen JH, Zhou XQ, Lu Q Jin LR, Huang Y. New-onset geriatric epilepsy in China: a single-center study. Chin Med J (Engl) 2018;131(24):2915-2920

11 Hauser WA, Annegers JF, Kurland LT. Incidence of epilepsy and unprovoked seizures in Rochester, Minnesota: 1935-1984. Epilepsia 1993;34(3):453-468

12 Loiseau J, Loiseau P, Duché B, Guyot M, Dartigues JF, Aublet B. A survey of epileptic disorders in southwest France: seizures in elderly patients. Ann Neurol 1990;27(3):232-237

13 Granger N, Convers P, Beauchet O, et al. First epileptic seizure in the elderly: electroclinical and etiological data in 341 patients. Rev Neurol 2002;158:1088-1095 